\title{
State-Feedback Stabilization for a Class of Stochastic Feedforward Nonlinear Time-Delay Systems
}

\author{
Liang Liu, ${ }^{1}$ Zhandong Yu, ${ }^{1}$ Qi Zhou, ${ }^{2}$ and Hamid Reza Karimi ${ }^{3}$ \\ ${ }^{1}$ College of Engineering, Bohai University, Liaoning 121013, China \\ ${ }^{2}$ College of Information Science, Bohai University, Liaoning 121013, China \\ ${ }^{3}$ Department of Engineering, Faculty of Engineering and Science, University of Agder, 4898 Grimstad, Norway
}

Correspondence should be addressed to Liang Liu; smithll@163.com

Received 18 October 2013; Accepted 18 November 2013

Academic Editor: Ming Liu

Copyright (c) 2013 Liang Liu et al. This is an open access article distributed under the Creative Commons Attribution License, which permits unrestricted use, distribution, and reproduction in any medium, provided the original work is properly cited.

We investigate the state-feedback stabilization problem for a class of stochastic feedforward nonlinear time-delay systems. By using the homogeneous domination approach and choosing an appropriate Lyapunov-Krasovskii functional, the delay-independent statefeedback controller is explicitly constructed such that the closed-loop system is globally asymptotically stable in probability. A simulation example is provided to demonstrate the effectiveness of the proposed design method.

\section{Introduction}

In recent years, the study on stochastic lower-triangular nonlinear systems has received considerable attention from both theoretical and practical point of views see, for instance, [1-19] and the references therein. This paper will further consider the following stochastic feedforward nonlinear timedelay systems described by

$$
\begin{aligned}
d x_{1}= & x_{2} d t+f_{1}\left(\tilde{x}_{3}, \tilde{x}_{3}(t-d(t))\right) d t \\
& +g_{1}^{T}\left(\tilde{x}_{2}, \widetilde{x}_{2}(t-d(t))\right) d \omega, \\
\vdots & \\
d x_{n-2}= & x_{n-1} d t+f_{n-2}\left(\tilde{x}_{n}, \widetilde{x}_{n}(t-d(t))\right) d t \\
& +g_{n-2}^{T}\left(\widetilde{x}_{n-1}, \tilde{x}_{n-1}(t-d(t))\right) d \omega, \\
d x_{n-1}= & x_{n} d t+g_{n-1}^{T}\left(\tilde{x}_{n}, \widetilde{x}_{n}(t-d(t))\right) d \omega, \\
d x_{n}= & u d t,
\end{aligned}
$$

where $x=\left(x_{1}, \ldots, x_{n}\right)^{T} \in R^{n}$ and $u \in R$ are the system state and input signal, respectively, $\widetilde{x}_{i}=\left(x_{i}, \ldots, x_{n}\right)^{T}, \widetilde{x}_{i}(t-d(t))=$ $\left(x_{i}(t-d(t)), \ldots, x_{n}(t-d(t))\right)^{T}$ is the time-delayed state vector, and $d(t): R_{+} \rightarrow[0, d]$ is the time-varying delay. $\omega$ is an $m$-dimensional standard Wiener process defined on the complete probability space $\left(\Omega, \mathscr{F},\left\{\mathscr{F}_{t}\right\}_{t \geq 0}, P\right)$ with $\Omega$ being a sample space, $\mathscr{F}$ being a $\sigma$-field, $\left\{\mathscr{F}_{t}\right\}_{t \geq 0}$ being a filtration, and $P$ being a probability measure. $f_{i}: R^{n-i-1} \times R^{n-i-1} \rightarrow R$ and $g_{j}: R^{n-j} \times R^{n-j} \rightarrow R^{m}$ are assumed to be locally Lipschitz with $f_{i}(0,0)=0$ and $g_{j}(0,0)=0, i=1, \ldots, n-2$, $j=1, \ldots, n-1$.

Feedforward (also called upper-triangular) system is another important class of nonlinear systems. Firstly, from a theoretical point of view, since they are not feedback linearizable and maybe not stabilized by applying the conventional backstepping method, the stabilization problem of these systems is more difficult than that of lower-triangular systems. Secondly, many physical devices, such as the cart-pendulum system in [20] and the ball-beam system with a friction term in [21], can be described by equations with the feedforward structure. In the recent papers, the stabilization problems for feedforward nonlinear (or time-delay) systems have achieved remarkable development; see, for example, [22-29] and the references therein.

However, all these above-mentioned results are limited to deterministic systems. There are fewer results on stochastic feedforward nonlinear systems until now, due to the special 
characteristics of this system. To the best of the authors' knowledge, [30] is the only paper to consider this kind of stochastic feedforward nonlinear systems, but the assumptions on the nonlinearities are restrictive.

The purpose of this paper is to further weaken the assumptions on the drift and diffusion terms of system (1) and solve the state-feedback stabilization problem. By using the homogeneous domination approach in [26] and choosing an appropriate Lyapunov-Krasovskii functional, a delay-independent state-feedback controller is explicitly constructed such that the closed-loop system is globally asymptotically stable in probability.

The paper is organized as follows. Section 2 provides some preliminary results. The design and analysis of statefeedback controller are given in Sections 3 and 4, respectively, following a simulation example in Section 5. Section 6 concludes this paper.

\section{Preliminary Results}

The following notations, definitions, and lemmas are to be used throughout the paper.

$R_{+}$denotes the set of all nonnegative real numbers, and $R^{n}$ denotes the real $n$-dimensional space. For a given vector or matrix $X, X^{T}$ denotes its transpose, $\operatorname{Tr}\{X\}$ denotes its trace when $X$ is square, and $|X|$ is the Euclidean norm of a vector $X . \mathscr{C}\left([-d, 0] ; R^{n}\right)$ denotes the space of continuous $R^{n}$-value functions on $[-d, 0]$ endowed with the norm $\|\cdot\|$ defined by $\|f\|=\sup _{x \in[-d, 0]}|f(x)|$ for $f \in \mathscr{C}\left([-d, 0] ; R^{n}\right)$; $\mathscr{C}_{\mathscr{F}_{0}}^{b}\left([-d, 0] ; R^{n}\right)$ denotes the family of all $\mathscr{F}_{0}$-measurable bounded $\mathscr{C}\left([-d, 0] ; R^{n}\right)$-valued random variables $\xi=\{\xi(\theta)$ : $-d \leq \theta \leq 0\} . \mathscr{C}^{i}$ denotes the set of all functions with continuous $i$ th partial derivatives; $\mathscr{C}^{2,1}\left(R^{n} \times[-d, \infty) ; R_{+}\right)$ denotes the family of all nonnegative functions $V(x, t)$ on $R^{n} \times[-d, \infty)$ which are $\mathscr{C}^{2}$ in $x$ and $\mathscr{C}^{1}$ in $t ; \mathscr{C}^{2,1}$ denotes the family of all functions which are $\mathscr{C}^{2}$ in the first argument and $\mathscr{C}^{1}$ in the second argument. $\mathscr{K}$ denotes the set of all functions $R_{+} \rightarrow R_{+}$, which are continuous, strictly increasing, and vanishing at zero; $\mathscr{K}_{\infty}$ denotes the set of all functions which are of class $\mathscr{K}$ and unbounded; $\mathscr{K} \mathscr{L}$ is the set of all functions $\beta(s, t): R_{+} \times R_{+} \rightarrow R_{+}$, which are of $\mathscr{K}$ for each fixed $t$ and decrease to zero as $t \rightarrow \infty$ for each fixed $s$.

Consider the following stochastic time-delay system:

$$
\begin{array}{r}
d x(t)=f(x(t), x(t-d(t)), t) d t \\
+g(x(t), x(t-d(t)), t) d \omega, \\
\forall t \geq 0,
\end{array}
$$

with initial data $\{x(\theta):-d \leq \theta \leq 0\}=\xi \in \mathscr{C}_{\mathscr{F}_{0}}^{b}\left([-d, 0] ; R^{n}\right)$, where $d(t): R_{+} \rightarrow[0, d]$ is a Borel measurable function, $\omega$ is an $m$-dimensional standard Wiener process defined on the complete probability space $\left(\Omega, \mathscr{F},\left\{\mathscr{F}_{t}\right\}_{t \geq 0}, P\right)$, and $f: R^{n} \times$ $R^{n} \times R_{+} \rightarrow R^{n}$ and $g: R^{n} \times R^{n} \times R_{+} \rightarrow R^{n \times m}$ are locally Lipschitz in $(x(t), x(t-d(t)))$ uniformly in $t$ with $f(0,0, t) \equiv 0$ and $g(0,0, t) \equiv 0$.
Definition 1 (see [6]). For any given $V(x(t), t) \in \mathscr{C}^{2,1}$ associated with system (2), the differential operator $\mathscr{L}$ is defined as

$$
\mathscr{L} V=\frac{\partial V}{\partial t}+\frac{\partial V}{\partial x} f+\frac{1}{2} \operatorname{Tr}\left\{g^{T} \frac{\partial^{2} V}{\partial x^{2}} g\right\} .
$$

Definition 2 (see [6]). The equilibrium $x(t)=0$ of system (2) is said to be globally asymptotically stable (GAS) in probability if for any $\epsilon>0$ there exists a function $\beta(\cdot, \cdot) \epsilon$ $\mathscr{K} \mathscr{L}$ such that $P\{|x(t)| \leq \beta(\|\xi\|, t)\} \geq 1-\epsilon$ for any $t \geq 0$, $\xi \in \mathscr{C}_{\mathscr{F}_{0}}^{b}\left([-d, 0] ; R^{n}\right) \backslash\{0\}$, where $\|\xi\|=\sup _{\theta \in[-d, 0]}|x(\theta)|$.

Definition 3 (see [26]). For fixed coordinates $\left(x_{1}, \ldots, x_{n}\right)^{T} \in$ $R^{n}$ and real numbers $r_{i}>0, i=1, \ldots, n$, one has the following.

(i) The dilation $\Delta_{\varepsilon}(x)$ is defined by $\Delta_{\varepsilon}(x)=$ $\left(\varepsilon^{r_{1}} x_{1}, \ldots, \varepsilon^{r_{n}} x_{n}\right)$ for any $\varepsilon>0 ; r_{1}, \ldots, r_{n}$ are called as the weights of the coordinates. For simplicity, we define dilation weight $\Delta=\left(r_{1}, \ldots, r_{n}\right)$.

(ii) A function $V \in \mathscr{C}\left(R^{n}, R\right)$ is said to be homogeneous of degree $\tau$ if there is a real number $\tau \in R$ such that $V\left(\Delta_{\varepsilon}(x)\right)=\varepsilon^{\tau} V\left(x_{1}, \ldots, x_{n}\right)$ for any $x \in R^{n} \backslash\{0\}, \varepsilon>0$.

(iii) A vector field $h \in \mathscr{C}\left(R^{n}, R^{n}\right)$ is said to be homogeneous of degree $\tau$ if there is a real number $\tau \in R$ such that $h_{i}\left(\Delta_{\varepsilon}(x)\right)=\varepsilon^{\tau+r_{i}} h_{i}(x)$ for any $x \in R^{n} \backslash\{0\}, \varepsilon>0$, $i=1, \ldots, n$.

(iv) A homogeneous $p$-norm is defined as $\|x\|_{\Delta, p}=$ $\left(\sum_{i=1}^{n}\left|x_{i}\right|^{p / r_{i}}\right)^{1 / p}$ for any $x \in R^{n}$, where $p \geq 1$ is a constant. For simplicity, in this paper, one chooses $p=2$ and writes $\|x\|_{\Delta}$ for $\|x\|_{\Delta, 2}$.

Lemma 4 (see [6]). For system (2), if there exist a function $V(x(t), t) \in \mathscr{C}^{2,1}\left(R^{n} \times[-d, \infty) ; R_{+}\right)$, two class $\mathscr{K}_{\infty}$ functions $\alpha_{1}, \alpha_{2}$, and a class $\mathscr{K}$ function $\alpha_{3}$ such that

$$
\begin{gathered}
\alpha_{1}(|x(t)|) \leq V(x(t), t) \leq \alpha_{2}\left(\sup _{-d \leq s \leq 0}|x(t+s)|\right), \\
\mathscr{L} V(x(t), t) \leq-\alpha_{3}(|x(t)|),
\end{gathered}
$$

then there exists a unique solution on $[-d, \infty)$ for (2), the equilibrium $x(t)=0$ is GAS in probability, and $P\left\{\lim _{t \rightarrow \infty}|x(t)|=\right.$ $0\}=1$.

Lemma 5 (see [26]). Given a dilation weight $\Delta=\left(r_{1}, \ldots, r_{n}\right)$, suppose that $V_{1}(x)$ and $V_{2}(x)$ are homogeneous functions of degrees $\tau_{1}$ and $\tau_{2}$, respectively. Then $V_{1}(x) V_{2}(x)$ is also homogeneous with respect to the same dilation weight $\Delta$. Moreover, the homogeneous degree of $V_{1} \cdot V_{2}$ is $\tau_{1}+\tau_{2}$.

Lemma 6 (see [26]). Suppose that $V: R^{n} \rightarrow R$ is a homogeneous function of degree $\tau$ with respect to the dilation weight $\Delta$; then (i) $\partial V / \partial x_{i}$ is homogeneous of degree $\tau-r_{i}$ with $r_{i}$ being the homogeneous weight of $x_{i}$; (ii) there is a constant $c$ such that $V(x) \leq c\|x\|_{\Delta}^{\tau}$. Moreover, if $V(x)$ is positive definite, then $V(x) \geq \underline{c}\|x\|_{\Delta}^{\tau}$, where $\underline{c}$ is a positive constant. 
Lemma 7 (see [5]). Let $c$ and $d$ be positive constants. For any positive number $\bar{\gamma}$, then $|x|^{c}|y|^{d} \leq(c /(c+d)) \bar{\gamma}|x|^{c+d}+(d /(c+$ d)) $\bar{\gamma}^{-c / d}|y|^{c+d}$.

\section{Design of State-Feedback Controller}

The objective of this paper is to design a state-feedback controller for system (1) such that the equilibrium of the closedloop system is globally asymptotically stable in probability.

3.1. Assumptions. For system (1), we need the following assumptions.

Assumption 8. For $i=1, \ldots, n-1$, there exist positive constants $a_{1}$ and $a_{2}$ such that

$$
\begin{aligned}
& \left|f_{i}\left(\tilde{x}_{i+2}, \tilde{x}_{i+2}(t-d(t))\right)\right| \\
& \quad \leq a_{1}\left(\sum_{j=i+2}^{n}\left|x_{j}\right|+\sum_{j=i+2}^{n}\left|x_{j}(t-d(t))\right|\right), \\
& \left|g_{i}\left(\tilde{x}_{i+1}, \tilde{x}_{i+1}(t-d(t))\right)\right| \\
& \quad \leq a_{2}\left(\sum_{j=i+1}^{n}\left|x_{j}\right|+\sum_{j=i+1}^{n}\left|x_{j}(t-d(t))\right|\right),
\end{aligned}
$$

where $x_{n+1}=x_{n+1}(t-d(t))=0$.

Assumption 9. The time-varying delay $d(t)$ satisfies $\dot{d}(t) \leq$ $\gamma<1$ for a constant $\gamma$.

Remark 10. When $x_{i+1}=x_{i+1}(t-d(t))=0$ in diffusion term $g_{i}(i=1, \ldots, n-1)$, Assumption 8 reduces to the same form as in [30], from which one can see that system (1) is more general than [30]. The significance and reasonability of Assumption 8 are illustrated in that paper.

Firstly, we introduce the following coordinate transformation:

$$
\eta_{i}=\frac{x_{i}}{\kappa^{i-1}}, \quad v=\frac{u}{\kappa^{n}}, \quad i=1, \ldots, n,
$$

where $0<\kappa<1$ is a scalar to be designed. By (6), (1) can be expressed as

$$
\begin{aligned}
d \eta_{1}= & \kappa \eta_{2} d t+\bar{f}_{1}\left(\widetilde{\eta}_{3}, \widetilde{\eta}_{3}(t-d(t))\right) d t \\
& +\bar{g}_{1}^{T}\left(\widetilde{\eta}_{2}, \widetilde{\eta}_{2}(t-d(t))\right) d \omega, \\
\vdots & \\
d \eta_{n-2}= & \kappa \eta_{n-1} d t+\bar{f}_{n-2}\left(\widetilde{\eta}_{n}, \widetilde{\eta}_{n}(t-d(t))\right) d t \\
& +\bar{g}_{n-2}^{T}\left(\widetilde{\eta}_{n-1}, \widetilde{\eta}_{n-1}(t-d(t))\right) d \omega, \\
d \eta_{n-1}= & \kappa \eta_{n} d t+\bar{g}_{n-1}^{T}\left(\widetilde{\eta}_{n}, \widetilde{\eta}_{n}(t-d(t))\right) d \omega, \\
d \eta_{n}= & \kappa v d t,
\end{aligned}
$$

where $\bar{f}_{i}=f_{i} / \kappa^{i-1}, \bar{g}_{i}=g_{i} / \kappa^{i-1}, i=1, \ldots, n-1, \bar{f}_{n-1}=0$.
3.2. State-Feedback Controller Design. We construct a statefeedback controller for system (7).

Step 1. Introducing $\xi_{1}=\eta_{1}$ and choosing $V_{1}\left(\eta_{1}\right)=(1 / 4) \xi_{1}^{4}$, from (3) and (7), it follows that

$$
\mathscr{L} V_{1}=\kappa \xi_{1}^{3} \eta_{2}+\frac{\partial V_{1}}{\partial \eta_{1}} \bar{f}_{1}+\frac{1}{2} \operatorname{Tr}\left\{\bar{g}_{1} \frac{\partial^{2} V_{1}}{\partial \eta_{1}^{2}} \bar{g}_{1}^{T}\right\} .
$$

The first virtual controller

$$
\eta_{2}^{*}=-c_{11} \xi_{1}=:-\alpha_{1} \xi_{1}, \quad c_{11}>0,
$$

leads to $\mathscr{L} V_{1} \leq-\kappa c_{11} \xi_{1}^{4}+\kappa \xi_{1}^{3}\left(\eta_{2}-\eta_{2}^{*}\right)+\left(\partial V_{1} / \partial \eta_{1}\right) \bar{f}_{1}+$ $(1 / 2) \operatorname{Tr}\left\{\bar{g}_{1}\left(\partial^{2} V_{1} / \partial \eta_{1}^{2}\right) \bar{g}_{1}^{T}\right\}$.

Step $i(i=2, \ldots, n)$. In this step, we can get the following lemma.

Lemma 11. Suppose that at step $i-1$, there is a set of virtual controllers $\eta_{1}^{*}, \ldots, \eta_{i}^{*}$ defined by

$$
\begin{gathered}
\eta_{1}^{*}=0, \quad \xi_{1}=\eta_{1}-\eta_{1}^{*}=\eta_{1}, \\
\eta_{k}^{*}=-\alpha_{k-1} \xi_{k-1}, \quad \xi_{k}=\eta_{k}-\eta_{k}^{*}, \quad k=2, \ldots, i,
\end{gathered}
$$

such that the $(i-1)$ th Lyapunov function $V_{i-1}\left(\bar{\eta}_{i-1}\right)=$ $(1 / 4) \sum_{j=1}^{i-1} \xi_{j}^{4}$ satisfies

$$
\begin{aligned}
\mathscr{L} V_{i-1} \leq & -\kappa \sum_{j=1}^{i-1} c_{i-1, j} \xi_{j}^{4}+\kappa \xi_{i-1}^{3}\left(\eta_{i}-\eta_{i}^{*}\right) \\
& +\sum_{j=1}^{i-1} \frac{\partial V_{i-1}}{\partial \eta_{j}} \bar{f}_{j}+\frac{1}{2} \sum_{p, q=1}^{i-1} \operatorname{Tr}\left\{\bar{g}_{p} \frac{\partial^{2} V_{i-1}}{\partial \eta_{p} \partial \eta_{q}} \bar{g}_{q}^{T}\right\},
\end{aligned}
$$

where $\alpha_{j}, c_{i-1, j}, j=1, \ldots, i-1$, are positive constants. Then there exists a virtual control law $\eta_{i+1}^{*}=-\alpha_{i} \xi_{i}$ such that

$$
\begin{aligned}
\mathscr{L} V_{i} \leq & -\kappa \sum_{j=1}^{i} c_{i j} \xi_{j}^{4}+\kappa \xi_{i}^{3}\left(\eta_{i+1}-\eta_{i+1}^{*}\right) \\
& +\sum_{j=1}^{i} \frac{\partial V_{i}}{\partial \eta_{j}} \bar{f}_{j}+\frac{1}{2} \sum_{p, q=1}^{i} \operatorname{Tr}\left\{\bar{g}_{p} \frac{\partial^{2} V_{i}}{\partial \eta_{p} \partial \eta_{q}} \bar{g}_{q}^{T}\right\},
\end{aligned}
$$

where $V_{i}\left(\bar{\eta}_{i}\right)=(1 / 4) \sum_{j=1}^{i} \xi_{j}^{4}=: V_{i-1}\left(\bar{\eta}_{i-1}\right)+W_{i}\left(\bar{\eta}_{i}\right)$.

Proof. See the Appendix.

$$
\begin{aligned}
& \text { At step } n \text {, choosing } V_{n}\left(\bar{\eta}_{n}\right)=(1 / 4) \sum_{i=1}^{n} \xi_{i}^{4} \text { and } \\
& \qquad \begin{aligned}
v & =\eta_{n+1}^{*}=-\alpha_{n} \xi_{n} \\
& =-\left(\bar{\alpha}_{n} \eta_{n}+\bar{\alpha}_{n-1} \eta_{n-1}+\cdots+\bar{\alpha}_{1} \eta_{1}\right),
\end{aligned}
\end{aligned}
$$


with the help of (3), (12), and (13), one obtains

$$
\begin{aligned}
\mathscr{L} V_{n} \leq & -\kappa \sum_{i=1}^{n} c_{n i} \xi_{i}^{4}+\kappa \xi_{n}^{3}\left(v-\eta_{n+1}^{*}\right) \\
& +\sum_{i=1}^{n} \frac{\partial V_{n}}{\partial \eta_{i}} \bar{f}_{i}+\frac{1}{2} \sum_{p, q=1}^{n} \operatorname{Tr}\left\{\bar{g}_{p} \frac{\partial^{2} V_{n}}{\partial \eta_{p} \partial \eta_{q}} \bar{g}_{q}^{T}\right\} \\
= & -\kappa \sum_{i=1}^{n} c_{n i} \xi_{i}^{4}+\frac{\partial V_{n}}{\partial \eta} F+\frac{1}{2} \operatorname{Tr}\left\{G \frac{\partial^{2} V_{n}}{\partial \eta^{2}} G^{T}\right\},
\end{aligned}
$$

where $F=\left(\bar{f}_{1}, \ldots, \bar{f}_{n-2}, 0,0\right)^{T}, G=\left(\bar{g}_{1}, \ldots, \bar{g}_{n-1}, 0\right), \xi_{n}=$ $\eta_{n}-\eta_{n}^{*}, \bar{\alpha}_{i}=\alpha_{n} \cdots \alpha_{i}, c_{n i}, i=1, \ldots, n$, are positive constants. The system (7) and (13) can be written as

$$
\begin{aligned}
d \eta= & \kappa E(\eta) d t+F(\eta, \eta(t-d(t))) d t \\
& +G^{T}(\eta, \eta(t-d(t))) d \omega,
\end{aligned}
$$

where $\eta=\bar{\eta}_{n}=\left(\eta_{1}, \ldots, \eta_{n}\right)^{T}, E(\eta)=\left(\eta_{2}, \ldots, \eta_{n}, v\right)^{T}$, and $F$ and $G$ are defined as in (14). Introducing the dilation weight $\Delta=(\underbrace{1,1, \ldots, 1}_{\text {for } \eta_{1}, \ldots, \eta_{n}})$, by $(10)$ and $V_{n}(\eta)=(1 / 4) \sum_{i=1}^{n} \xi_{i}^{4}$, one has

$$
\begin{aligned}
V_{n} & \left(\Delta_{\varepsilon}(\eta)\right) \\
& =\frac{1}{4} \sum_{i=1}^{n}\left(\varepsilon \eta_{i}+\alpha_{i-1} \varepsilon \eta_{i-1}+\cdots+\alpha_{i-1} \cdots \alpha_{1} \varepsilon \eta_{1}\right)^{4} \\
& =\varepsilon^{4} V_{n}(\eta),
\end{aligned}
$$

from which and Definition 3, we know that $V_{n}(\eta)$ is homogeneous of degree 4.

\section{Stability Analysis}

We state the main result in this paper.

Theorem 12. If Assumptions 8 and 9 hold for the stochastic feedforward nonlinear time-delay system (1), under the statefeedback controller $u=\kappa^{n} v$ and (13), then

(i) the closed-loop system has a unique solution on $[-d, \infty)$;

(ii) the equilibrium at the origin of the closed-loop system is GAS in probability.

Proof. We prove Theorem 12 by four steps.

Step 1 . Since $f_{i}, g_{i}, i=1, \ldots, n$, are assumed to be locally Lipschitz, so the system consisting of (13) and (15) satisfies the locally Lipschitz condition.

Step 2. We consider the following entire Lyapunov function for system (15):

$$
V(\eta)=V_{n}(\eta)+\frac{\left(\bar{c}_{02}+\bar{c}_{03}\right) \kappa^{2}}{1-\gamma} \int_{t-d(t)}^{t}\|\eta(\sigma)\|_{\Delta}^{4} d \sigma,
$$

where $\bar{c}_{02}$ and $\bar{c}_{03}$ are positive parameters to be determined. It is easy to verify that $V(\eta)$ is $\mathscr{C}^{2}$ on $\eta$. Since $V_{n}(\eta)$ is continuous, positive definite, and radially unbounded, by Lemma 4.3 in [31], there exist two class $\mathscr{K}_{\infty}$ functions $\beta_{1}$ and $\alpha_{21}$ such that

$$
\beta_{1}(|\eta|) \leq V_{n}(\eta) \leq \alpha_{21}(|\eta|)
$$

By Lemma 4.3 in [31] and Lemma 6, there exist positive constants $\underline{c}$ and $\bar{c}$, class $\mathscr{K}_{\infty}$ functions $\underline{\alpha}_{22}$ and $\bar{\alpha}_{22}$, and a positive definite function $U(\eta)$ whose homogeneous degree is 4 such that

$$
\begin{gathered}
\underline{c}\|\eta\|_{\Delta}^{4} \leq U(\eta) \leq \bar{c}\|\eta\|_{\Delta}^{4}, \\
\underline{\alpha}_{22}(|\eta|) \leq U(\eta) \leq \bar{\alpha}_{22}(|\eta|) .
\end{gathered}
$$

From $d(t): R_{+} \rightarrow[0, d]$ and (19), it follows that

$$
\begin{gathered}
\frac{\left(\bar{c}_{02}+\bar{c}_{03}\right) \kappa^{2}}{1-\gamma} \int_{t-d(t)}^{t}\|\eta(\sigma)\|_{\Delta}^{4} d \sigma \\
\leq \widetilde{c} \int_{t-d(t)}^{t} \bar{\alpha}_{22}(|\eta(\sigma)|) d \sigma \\
\stackrel{\sigma=s+t}{=} \tilde{c} \int_{-d(t)}^{0} \bar{\alpha}_{22}(|\eta(s+t)|) d(s+t) \\
\leq \widetilde{c} \int_{-d}^{0} \bar{\alpha}_{22}(|\eta(s+t)|) d(s+t) \\
\leq c \sup _{-d \leq s \leq 0} \bar{\alpha}_{22}(|\eta(s+t)|) \\
\leq \alpha_{22}\left(\sup _{-d \leq s \leq 0}|\eta(s+t)|\right)
\end{gathered}
$$

where $\tilde{c}, c$ are positive constants and $\alpha_{22}$ is a class $\mathscr{K}_{\infty}$ function. Since $|\eta| \leq \sup _{-d \leq s \leq 0}|\eta(s+t)|, \alpha_{21}(|\eta|) \leq$ $\alpha_{21}\left(\sup _{-d \leq s \leq 0}|\eta(s+t)|\right)$. Defining $\beta_{2}=\alpha_{21}+\alpha_{22}$, by (17), (18), and (20), one gets

$$
\beta_{1}(|\eta|) \leq V(\eta) \leq \beta_{2}\left(\sup _{-d \leq s \leq 0}|\eta(s+t)|\right) .
$$

Step 3. By Lemma 6 and (14), there exists a positive constant $c_{01}$ such that

$$
\frac{\partial V_{n}}{\partial \eta} \kappa E(\eta) \leq-c_{01} \kappa\|\eta\|_{\Delta}^{4}
$$

By Assumption 8, (6), and $0<\kappa<1$, one has

$$
\begin{aligned}
& \left|\bar{f}_{i}\left(\tilde{\eta}_{i+2}, \tilde{\eta}_{i+2}(t-d(t))\right)\right| \\
& \leq \frac{a_{1}\left(\sum_{j=i+2}^{n}\left|\kappa^{j-1} \eta_{j}\right|+\sum_{j=i+2}^{n}\left|\kappa^{j-1} \eta_{j}(t-d(t))\right|\right)}{\kappa^{i-1}} \\
& \quad \leq a_{1} \kappa^{2}\left(\sum_{j=i+2}^{n}\left|\eta_{j}\right|+\sum_{j=i+2}^{n}\left|\eta_{j}(t-d(t))\right|\right) \\
& \quad \leq \delta_{1} \kappa^{2}\left(\|\eta\|_{\Delta}+\|\eta(t-d(t))\|_{\Delta}\right)
\end{aligned}
$$


where $\delta_{1}$ is a positive constant. Using Lemmas 5-7 and (23), one gets

$$
\begin{aligned}
& \frac{\partial V_{n}}{\partial \eta} F(\eta, \eta(t-d(t))) \\
& \quad=\sum_{i=1}^{n-2} \frac{\partial V_{n}}{\partial \eta_{i}} \bar{f}_{i}\left(\widetilde{\eta}_{i+2}, \widetilde{\eta}_{i+2}(t-d(t))\right) \\
& \quad \leq \widetilde{c}_{02} \kappa^{2} \sum_{i=1}^{n-2}\|\eta\|_{\Delta}^{3}\left(\|\eta\|_{\Delta}+\|\eta(t-d(t))\|_{\Delta}\right) \\
& \quad \leq \kappa^{2}\left(c_{02}\|\eta\|_{\Delta}^{4}+\bar{c}_{02}\|\eta(t-d(t))\|_{\Delta}^{4}\right),
\end{aligned}
$$

where $c_{02}, \bar{c}_{02}$, and $\widetilde{c}_{02}$ are positive constants. Similar to (23), there is a positive constant $\delta_{2}$ such that

$$
\left|\bar{g}_{i}\left(\widetilde{\eta}_{i+1}, \widetilde{\eta}_{i+1}(t-d(t))\right)\right| \leq \delta_{2} \kappa\left(\|\eta\|_{\Delta}+\|\eta(t-d(t))\|_{\Delta}\right),
$$

from which and Lemmas 5-7, one gets

$$
\begin{aligned}
& \frac{1}{2} \operatorname{Tr}\left\{G(\eta, \eta(t-d(t))) \frac{\partial^{2} V_{n}}{\partial \eta^{2}} G^{T}(\eta, \eta(t-d(t)))\right\} \\
& \leq \frac{1}{2} m \sqrt{m} \sum_{i, j=1}^{n-1}\left|\frac{\partial^{2} V_{n}}{\partial \eta_{i} \partial \eta_{j}}\right|\left|\bar{g}_{i}\left(\widetilde{\eta}_{i+1}, \widetilde{\eta}_{i+1}(t-d(t))\right)\right| \\
& \quad \times\left|\bar{g}_{j}\left(\widetilde{\eta}_{j+1}, \widetilde{\eta}_{j+1}(t-d(t))\right)\right| \\
& \leq \widetilde{c}_{03} \kappa^{2} \sum_{i, j=1}^{n-1}\|\eta\|_{\Delta}^{2}\left(\|\eta\|_{\Delta}+\|\eta(t-d(t))\|_{\Delta}\right)^{2} \\
& \leq \kappa^{2}\left(c_{03}\|\eta\|_{\Delta}^{4}+\bar{c}_{03}\|\eta(t-d(t))\|_{\Delta}^{4}\right),
\end{aligned}
$$

where $c_{03}, \bar{c}_{03}$, and $\widetilde{c}_{03}$ are positive constants. With the help of (3), (15), (17), (22), (24), (26), and Assumption 9, one has

$$
\begin{aligned}
\mathscr{L} V \leq & \frac{\partial V_{n}}{\partial \eta} \kappa E(\eta)+\frac{\partial V_{n}}{\partial \eta} F(\eta, \eta(t-d(t))) \\
& +\frac{1}{2} \operatorname{Tr}\left\{G(\eta, \eta(t-d(t))) \frac{\partial^{2} V_{n}}{\partial \eta^{2}} G^{T}(\eta, \eta(t-d(t)))\right\} \\
& +\left(\bar{c}_{02}+\bar{c}_{03}\right) \kappa^{2}\left(\frac{1}{1-\gamma}\|\eta\|_{\Delta}^{4}-\|\eta(t-d(t))\|_{\Delta}^{4}\right) \\
\leq & -c_{01} \kappa\|\eta\|_{\Delta}^{4}+\left(c_{02}+c_{03}+\frac{\bar{c}_{02}+\bar{c}_{03}}{1-\gamma}\right) \kappa^{2}\|\eta\|_{\Delta}^{4} \\
= & -\kappa\left(c_{01}-\left(c_{02}+c_{03}+\frac{\bar{c}_{02}+\bar{c}_{03}}{1-\gamma}\right) \kappa\right)\|\eta\|_{\Delta}^{4} .
\end{aligned}
$$

Since $c_{01}$ is a constant independent of $c_{02}, c_{03}, \bar{c}_{02}, \bar{c}_{03}$, and $\gamma$, by choosing

$$
0<\kappa<\kappa^{*}=: \min \left\{1, \frac{c_{01}}{c_{02}+c_{03}+\left(\left(\bar{c}_{02}+\bar{c}_{03}\right) /(1-\gamma)\right)}\right\} .
$$

Equation (27) becomes $\mathscr{L} V \leq-c_{0}\|\eta\|_{\Delta}^{4}$, where $c_{0}$ is a positive constant. By (19), one obtains

$$
\mathscr{L} V \leq-\frac{\mathcal{c}_{0}}{\bar{c}} \underline{\alpha}_{22}(|\eta|) .
$$

By Steps 1-3 and Lemma 4, the system consisting of (13) and (15) has a unique solution on $[-d, \infty), \eta=0$ is GAS in probability, and $P\left\{\lim _{t \rightarrow \infty}|\eta|=0\right\}=1$.

Step 4. Since (6) is an equivalent transformation, so the closed-loop system consisting of (1), $u=\kappa^{n} v$, and (13) has the same properties as the system (13) and (15). Theorem 12 holds.

Remark 13. In this paper, the homogeneous domination idea is generalized to stochastic feedforward nonlinear time-delay systems (1). The underlying philosophy of this approach is that the state-feedback controller is first constructed for system (7) without considering the drift and diffusion terms, and then a low gain $\kappa$ in (6) (whose the value range is (28)) is introduced to state-feedback controller to dominate the drift and diffusion terms.

Remark 14. Due to the special upper-triangular structure and the appearance of time-varying delay, there is no efficient method to solve the stabilization problem of system (1). By combining the homogeneous domination approach with stochastic nonlinear time-delay system criterion, the statefeedback stabilization of system (1) was perfectly solved in this paper.

Remark 15. One of the main obstacles in the stability analysis is how to deal with the effect of time-varying delay. In this paper, by constructing an appropriate Lyapunov-Krasovskii functional (17), this problem was effectively solved.

Remark 16. It is worth pointing out that the rigorous proof of Theorem 12 is not an easy job.

\section{A Simulation Example}

Consider the following stochastic nonlinear system:

$$
\begin{gathered}
d x_{1}=x_{2} d t+\frac{1}{10}\left(x_{2}+x_{2}(t-d(t)) \cos x_{2}\right) d \omega, \\
d x_{2}=u d t,
\end{gathered}
$$

where $d(t)=1+(1 / 2) \sin t$. It is easy to verify that Assumptions 8 and 9 are satisfied with $a_{1}=0, a_{2}=1 / 10$, and $\dot{d}(t)=(1 / 2) \cos t<1$.

Design of Controller. Introducing the following coordinate transformation:

$$
\eta_{1}=x_{1}, \quad \eta_{2}=\frac{x_{2}}{\kappa}, \quad v=\frac{u}{\kappa^{2}},
$$

system (30) becomes

$$
\begin{gathered}
d \eta_{1}=\kappa \eta_{2} d t+\bar{g}_{1} d \omega, \\
d \eta_{2}=\kappa v d t,
\end{gathered}
$$




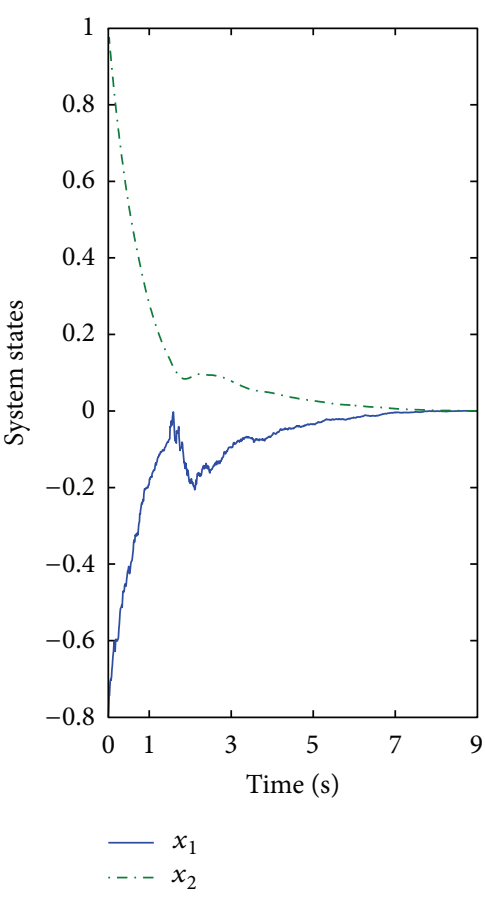

(a)

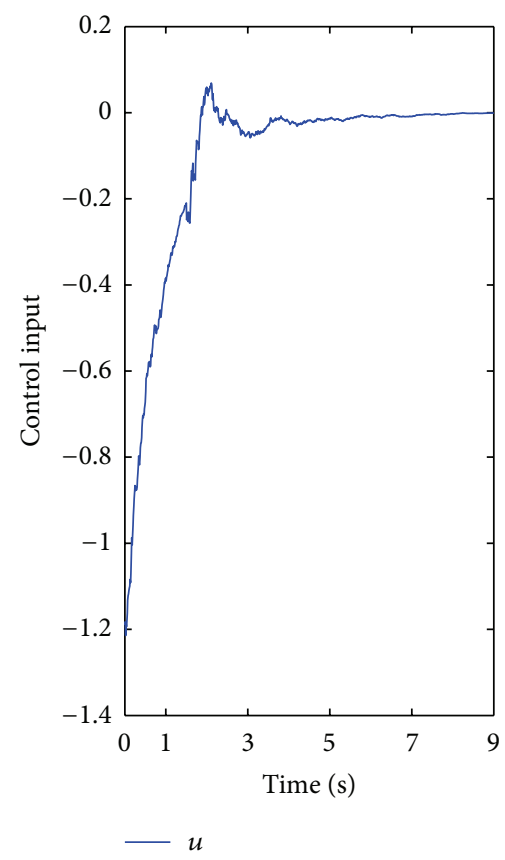

(b)

FIgURE 1: (a) The response of the closed-loop system (30) and (b) the response of the controller (37).

where $\bar{g}_{1}=(1 / 10)\left(\kappa \eta_{2}+\kappa \eta_{2}(t-d(t)) \cos \kappa \eta_{2}\right)$. Choosing $\xi_{1}=$ $\eta_{1}$ and $V_{1}\left(\eta_{1}\right)=(1 / 4) \xi_{1}^{4}$, we obtain $\mathscr{L} V_{1} \leq-2 \kappa \xi_{1}^{4}+\kappa \xi_{1}^{3}\left(\eta_{2}-\right.$ $\left.\eta_{2}^{*}\right)+(1 / 2)\left(\partial^{2} V_{1} / \partial \eta_{1}^{2}\right) \bar{g}_{1}^{2}$, where $\eta_{2}^{*}=-2 \eta_{1}=:-\alpha_{1} \xi_{1}$. By $\xi_{2}=$ $\eta_{2}-\eta_{2}^{*}$ and $V_{2}\left(\bar{\eta}_{2}\right)=V_{1}\left(\eta_{1}\right)+(1 / 4) \xi_{2}^{4}$, a direct calculation leads to

$$
\mathscr{L} V_{2} \leq-2 \kappa \xi_{1}^{4}+\kappa \xi_{1}^{3} \xi_{2}+\kappa \xi_{2}^{3} v+\kappa \alpha_{1} \xi_{2}^{3} \eta_{2}+\frac{1}{2} \frac{\partial^{2} V_{2}}{\partial \eta_{1}^{2}} \bar{g}_{1}^{2}
$$

By Lemma 7, one has

$$
\begin{gathered}
\xi_{1}^{3} \xi_{2} \leq 0.5 \xi_{1}^{4}+0.8438 \xi_{2}^{4}, \\
\alpha_{1} \xi_{2}^{3} \eta_{2} \leq 0.5 \xi_{1}^{4}+5.7797 \xi_{2}^{4} .
\end{gathered}
$$

Choosing

$$
v=-7.6235 \xi_{2}=:-\alpha_{2} \xi_{2}
$$

and substituting (34) into (33), one gets

$$
\mathscr{L} V_{2} \leq-\kappa\left(\xi_{1}^{4}+\xi_{2}^{4}\right)+\frac{1}{2} \frac{\partial^{2} V_{2}}{\partial \eta_{1}^{2}} \bar{g}_{1}^{2} .
$$

By (31) and (35), one obtains the actual controller

$$
u=-\alpha_{2}\left(\kappa x_{2}+\alpha_{1} \kappa^{2} x_{1}\right) .
$$

The Choice of $\kappa^{*}$. Defining $\|\eta\|_{\Delta}=\left(\eta_{1}^{2}+\eta_{2}^{2}\right)^{1 / 2}$ and choosing

$$
V(\eta)=V_{2}(\eta)+\kappa^{2} \int_{t-d(t)}^{t}\|\eta(\sigma)\|_{\Delta}^{4} d \sigma
$$

by (3), (36), and $d(t)=1+(1 / 2) \sin t$, one obtains

$$
\begin{aligned}
\mathscr{L} V \leq & -\kappa\|\eta\|_{\Delta}^{4}+\kappa^{2}\left(1.5\|\eta\|_{\Delta}^{4}+0.5\|\eta(t-d(t))\|_{\Delta}^{4}\right) \\
& +\kappa^{2}\left(\|\eta\|_{\Delta}^{4}-0.5\|\eta(t-d(t))\|_{\Delta}^{4}\right) \\
= & -\kappa(1-2.5 \kappa)\|\eta\|_{\Delta}^{4},
\end{aligned}
$$

from which we get the critical value $\kappa^{*}=0.4$; that is, $\kappa \in$ $(0,0.4)$.

In simulation, we choose the initial values $x_{1}(0)=$ $-0.8, x_{2}(0)=1$, and $\kappa=0.3$. Figure 1 demonstrates the effectiveness of the state-feedback controller.

\section{A Concluding Remark}

By using the homogeneous domination approach, this paper further studied the state-feedback stabilization problem for a class of stochastic feedforward nonlinear time-delay systems (1). The delay-independent state-feedback controller is explicitly constructed such that the closed-loop system is globally asymptotically stable in probability.

There still exist some problems to be investigated. One is to consider the output-feedback control of switched stochastic system (1) by using average dwell time method in [32]. Another is to find a practical example (similar to [3335]) for system (1). The last is to generalize the networked control systems (such as [36-41]) to stochastic feedforward networked systems. 


\section{Appendix}

Proof of Lemma 11. According to (3), (7), (10), and (11), one has

$$
\begin{aligned}
& \mathscr{L} V_{i} \leq-\kappa \sum_{j=1}^{i-1} c_{i-1, j} \xi_{j}^{4}+\kappa \xi_{i-1}^{3} \xi_{i}+\sum_{j=1}^{i-1} \frac{\partial V_{i-1}}{\partial \eta_{j}} \bar{f}_{j} \\
& +\frac{1}{2} \sum_{p, q=1}^{i-1} \operatorname{Tr}\left\{\bar{g}_{p} \frac{\partial^{2} V_{i-1}}{\partial \eta_{p} \partial \eta_{q}} \bar{g}_{q}^{T}\right\} \\
& +\sum_{k=1}^{i} \frac{\partial W_{i}}{\partial \eta_{k}}\left(\kappa \eta_{k+1}+\bar{f}_{k}\right)+\frac{1}{2} \operatorname{Tr}\left\{\bar{g}_{i} \frac{\partial^{2} W_{i}}{\partial \eta_{i}^{2}} \bar{g}_{i}^{T}\right\} \\
& +\sum_{j=1}^{i-1} \operatorname{Tr}\left\{\bar{g}_{i} \frac{\partial^{2} W_{i}}{\partial \eta_{i} \partial \eta_{j}} \bar{g}_{j}^{T}\right\}+\frac{1}{2} \sum_{p, q=1}^{i-1} \operatorname{Tr}\left\{\bar{g}_{p} \frac{\partial^{2} W_{i}}{\partial \eta_{p} \partial \eta_{q}} \bar{g}_{q}^{T}\right\} \\
& =-\kappa \sum_{j=1}^{i-1} c_{i-1, j} \xi_{j}^{4}+\kappa \xi_{i-1}^{3} \xi_{i}+\frac{\partial W_{i}}{\partial \eta_{i}} \kappa \eta_{i+1} \\
& +\left(\sum_{j=1}^{i-1} \frac{\partial V_{i-1}}{\partial \eta_{j}} \bar{f}_{j}+\sum_{k=1}^{i} \frac{\partial W_{i}}{\partial \eta_{k}} \bar{f}_{k}\right)+\sum_{k=1}^{i-1} \frac{\partial W_{i}}{\partial \eta_{k}} \kappa \eta_{k+1} \\
& +\left(\frac{1}{2} \sum_{p, q=1}^{i-1} \operatorname{Tr}\left\{\bar{g}_{p} \frac{\partial^{2} V_{i-1}}{\partial \eta_{p} \partial \eta_{q}} \bar{g}_{q}^{T}\right\}+\frac{1}{2} \operatorname{Tr}\left\{\bar{g}_{i} \frac{\partial^{2} V_{i}}{\partial \eta_{i}^{2}} \bar{g}_{i}^{T}\right\}\right. \\
& +\sum_{j=1}^{i-1} \operatorname{Tr}\left\{\bar{g}_{i} \frac{\partial^{2} V_{i}}{\partial \eta_{i} \partial \eta_{j}} \bar{g}_{j}^{T}\right\} \\
& \left.+\frac{1}{2} \sum_{p, q=1}^{i-1} \operatorname{Tr}\left\{\bar{g}_{p} \frac{\partial^{2} W_{i}}{\partial \eta_{p} \partial \eta_{q}} \bar{g}_{q}^{T}\right\}\right) \\
& =-\kappa \sum_{j=1}^{i-1} c_{i-1, j} \xi_{j}^{4}+\kappa \xi_{i}^{3} \eta_{i+1}+\sum_{j=1}^{i} \frac{\partial V_{i}}{\partial \eta_{j}} \bar{f}_{j} \\
& +\frac{1}{2} \sum_{p, q=1}^{i} \operatorname{Tr}\left\{\bar{g}_{p} \frac{\partial^{2} V_{i}}{\partial \eta_{p} \partial \eta_{q}} \bar{g}_{q}^{T}\right\} \\
& +\kappa \xi_{i-1}^{3} \xi_{i}-\kappa \xi_{i}^{3} \sum_{k=1}^{i-1} \frac{\partial \eta_{i}^{*}}{\partial \eta_{k}} \eta_{k+1}
\end{aligned}
$$

We concentrate on the last two terms on the right-hand side of (A.1)

Using (10) and Lemma 7, one obtains

$$
\begin{gathered}
\xi_{i-1}^{3} \xi_{i} \leq l_{i, i-1,1} \xi_{i-1}^{4}+\rho_{i 1} \xi_{i}^{4}, \\
-\xi_{i}^{3} \sum_{k=1}^{i-1} \frac{\partial \eta_{i}^{*}}{\partial \eta_{k}} \eta_{k+1} \\
\leq\left|\xi_{i}\right|^{3}\left|\sum_{k=1}^{i-1} \alpha_{i-1} \cdots \alpha_{k}\left(\xi_{k+1}-\alpha_{k} \xi_{k}\right)\right|
\end{gathered}
$$

$$
\begin{aligned}
& \leq\left|\xi_{i}\right|^{3}\left(\sum_{k=1}^{i-1}\left(\alpha_{i-1} \cdots \alpha_{k-1}+\alpha_{i-1} \cdots \alpha_{k+1} \alpha_{k}^{2}\right)\left|\xi_{k}\right|\right. \\
& \left.\quad+\alpha_{i-1}\left|\xi_{i}\right|\right) \\
& \leq \sum_{k=1}^{i-1} l_{i k 2} \xi_{k}^{4}+\rho_{i 2} \xi_{i}^{4},
\end{aligned}
$$

where $l_{i, i-1,1}, l_{i k 2}(k=1, \ldots, i-1), \rho_{i 1}$, and $\rho_{i 2}$ are positive constants, $\alpha_{0}=0$.

Choosing

$$
\begin{gathered}
c_{i j}= \begin{cases}c_{i-1, j}-l_{i j 2}>0, & j=1, \ldots, i-2, \\
c_{i-1, i-1}-l_{i, i-1,1}-l_{i, i-1,2}>0, & j=i-1,\end{cases} \\
\eta_{i+1}^{*}=-\left(c_{i i}+\rho_{i 1}+\rho_{i 2}\right) \xi_{i}=:-\alpha_{i} \xi_{i}, \quad c_{i i}>0,
\end{gathered}
$$

and substituting (A.2)-(A.3) into (A.1), one gets the desired result.

\section{Conflict of Interests}

The authors declare that there is no conflict of interests.

\section{Acknowledgments}

The authors would like to express sincere gratitude to editor and reviewers for their helpful suggestions in improving the quality of this paper. This work was partially supported by the National Natural Science Foundation of China (nos. 61304002, 61304003, 61203123, and 61304054), the Fundamental Research Funds for the Central Universities of China (no. 11CX04044A), the Shandong Provincial Natural Science Foundation of China (no. ZR2012FQ019), and the PolishNorwegian Research Programme operated by the National Center for Research and 24 Development under the Norwegian Financial Mechanism 2009-2014 in the frame of Project Contract (no. Pol-Nor/200957/47/2013).

\section{References}

[1] N. Duan and X.-J. Xie, "Further results on output-feedback stabilization for a class of stochastic nonlinear systems," IEEE Transactions on Automatic Control, vol. 56, no. 5, pp. 1208-1213, 2011.

[2] M. Krstić and H. Deng, Stabilization of Uncertain Nonlinear Systems, Springer, New York, NY, USA, 1998.

[3] S. Kuang, Y. Peng, F. Deng, and W. Gao, "Exponential stability and numerical methods of stochastic recurrent neural networks with delays," Abstract and Applied Analysis, vol. 2013, Article ID 761237, 11 pages, 2013.

[4] W.-Q. Li and X.-J. Xie, "Inverse optimal stabilization for stochastic nonlinear systems whose linearizations are not stabilizable," Automatica, vol. 45, no. 2, pp. 498-503, 2009.

[5] L. Liu and X.-J. Xie, "Output-feedback stabilization for stochastic high-order nonlinear systems with time-varying delay," Automatica, vol. 47, no. 12, pp. 2772-2779, 2011. 
[6] S.-J. Liu, S. S. Ge, and J.-F. Zhang, "Adaptive output-feedback control for a class of uncertain stochastic non-linear systems with time delays," International Journal of Control, vol. 81, no. 8, pp. 1210-1220, 2008.

[7] S.-J. Liu, J.-F. Zhang, and Z.-P. Jiang, "Decentralized adaptive output-feedback stabilization for large-scale stochastic nonlinear systems," Automatica, vol. 43, no. 2, pp. 238-251, 2007.

[8] Y.-G. Liu and J.-F. Zhang, "Practical output-feedback risksensitive control for stochastic nonlinear systems with stable zero-dynamics," SIAM Journal on Control and Optimization, vol. 45, no. 3, pp. 885-926, 2006.

[9] L. Wu, P. Shi, and H. Gao, "State estimation and slidingmode control of Markovian jump singular systems," IEEE Transactions on Automatic Control, vol. 55, no. 5, pp. 1213-1219, 2010.

[10] Z.-J. Wu, X.-J. Xie, and S.-Y. Zhang, "Adaptive backstepping controller design using stochastic small-gain theorem," Automatica, vol. 43, no. 4, pp. 608-620, 2007.

[11] Z.-J. Wu, X.-J. Xie, P. Shi, and Y.-Q. Xia, "Backstepping controller design for a class of stochastic nonlinear systems with Markovian switching," Automatica, vol. 45, no. 4, pp. 997-1004, 2009.

[12] X.-J. Xie and N. Duan, "Output tracking of high-order stochastic nonlinear systems with application to benchmark mechanical system," IEEE Transactions on Automatic Control, vol. 55, no. 5, pp. 1197-1202, 2010.

[13] X.-J. Xie and L. Liu, "Further results on output feedback stabilization for stochastic high-order nonlinear systems with time-varying delay," Automatica, vol. 48, no. 10, pp. 2577-2586, 2012.

[14] X.-J. Xie and L. Liu, "A homogeneous domination approach to state feedback of stochastic high-order nonlinear systems with time-varying delay," IEEE Transactions on Automatic Control, vol. 58, no. 2, pp. 494-499, 2013.

[15] X.-J. Xie and J. Tian, "Adaptive state-feedback stabilization of high-order stochastic systems with nonlinear parameterization," Automatica, vol. 45, no. 1, pp. 126-133, 2009.

[16] S. Yin, S. X. Ding, A. H. A. Sari, and H. Hao, "Data-driven monitoring for stochastic systems and its application on batch process," International Journal of Systems Science, vol. 44, no. 7, pp. 1366-1376, 2013.

[17] X. Yu and X.-J. Xie, "Output feedback regulation of stochastic nonlinear systems with stochastic iISS inverse dynamics," IEEE Transactions on Automatic Control, vol. 55, no. 2, pp. 304-320, 2010.

[18] X. Zhao, M. Ling, and Q. Zeng, "Delay-dependent robust control for uncertain stochastic systems with Markovian switching and multiple delays," Journal of Systems Engineering and Electronics, vol. 21, no. 2, pp. 287-295, 2010.

[19] X. Zhou, Y. Ren, and S. Zhong, "BIBO stabilization of discretetime stochastic control systems with mixed delays and nonlinear perturbations," Abstract and Applied Analysis, vol. 2013, Article ID 916357, 11 pages, 2013.

[20] F. Mazenc and S. Bowong, "Tracking trajectories of the cartpendulum system," Automatica, vol. 39, no. 4, pp. 677-684, 2003.

[21] R. Sepulchre, M. Janković, and P. V. Kokotović, Constructive Nonlinear Control, Springer, London, UK, 1997.

[22] N. Bekiaris-Liberis and M. Krstić, "Delay-adaptive feedback for linear feedforward systems," Systems \& Control Letters, vol. 59, no. 5, pp. 277-283, 2010.
[23] S. Ding, C. Qian, S. Li, and Q. Li, "Global stabilization of a class of upper-triangular systems with unbounded or uncontrollable linearizations," International Journal of Robust and Nonlinear Control, vol. 21, no. 3, pp. 271-294, 2011.

[24] A. Isidori, Nonlinear Control Systems, Springer, London, UK, 1999.

[25] M. Krstić, "Input delay compensation for forward complete and strict-feedforward nonlinear systems," IEEE Transactions on Automatic Control, vol. 55, no. 2, pp. 287-302, 2010.

[26] C. Qian and J. Li, "Global output feedback stabilization of upper-triangular nonlinear systems using a homogeneous domination approach," International Journal of Robust and Nonlinear Control, vol. 16, no. 9, pp. 441-463, 2006.

[27] X. Ye, "Adaptive stabilization of time-delay feedforward nonlinear systems," Automatica, vol. 47, no. 5, pp. 950-955, 2011.

[28] X. Zhang, L. Baron, Q. Liu, and E.-K. Boukas, "Design of stabilizing controllers with a dynamic gain for feedforward nonlinear time-delay systems," IEEE Transactions on Automatic Control, vol. 56, no. 3, pp. 692-697, 2011.

[29] X. Zhang, Q. Liu, L. Baron, and E.-K. Boukas, "Feedback stabilization for high order feedforward nonlinear time-delay systems," Automatica, vol. 47, no. 5, pp. 962-967, 2011.

[30] L. Liu and X.-J. Xie, "State feedback stabilization for stochastic feedforward nonlinear systems with time-varying delay," Automatica, vol. 49, no. 4, pp. 936-942, 2013.

[31] H. K. Khalil, Nonlinear Systems, Prentice-Hall, Upper Saddle River, NJ, USA, 3rd edition, 2002.

[32] L. Zhang and H. Gao, "Asynchronously switched control of switched linear systems with average dwell time," Automatica, vol. 46, no. 5, pp. 953-958, 2010.

[33] H. L. Dong, Z. D. Wang, and H. J. Gao, “Distributed $H_{\infty}$ filtering for a class of markovian jump nonlinear time-delay systems over lossy sensor networks," IEEE Transactions on Industrial Electronics, vol. 60, no. 10, pp. 4665-4672, 2013.

[34] X. C. Xie, S. Yin, H. J. Gao, and O. Kaynak, "Asymptotic stability and stabilisation of uncertain delta operator systems with timevarying delays," IET Control Theory and Applications, vol. 7, no. 8, pp. 1071-1078, 2013.

[35] S. Yin, S. X. Ding, and H. Luo, "Real-time implementation of fault tolerant control system with performance optimization," IEEE Transactions on Industrial Electronics, vol. 61, no. 5, pp. 2402-2411, 2014.

[36] H. Gao and T. Chen, " $H_{\infty}$ estimation for uncertain systems with limited communication capacity," IEEE Transactions on Automatic Control, vol. 52, no. 11, pp. 2070-2084, 2007.

[37] H. Gao, T. Chen, and J. Lam, "A new delay system approach to network-based control," Automatica, vol. 44, no. 1, pp. 39-52, 2008.

[38] H. Gao and T. Chen, "Network-based $H_{\infty}$ output tracking control," IEEE Transactions on Automatic Control, vol. 53, no. 3, pp. 655-667, 2008.

[39] H. Gao, X. Meng, and T. Chen, "Stabilization of networked control systems with a new delay characterization," IEEE Transactions on Automatic Control, vol. 53, no. 9, pp. 2142-2148, 2008.

[40] H. Li, H. Gao, and P. Shi, "New passivity analysis for neural networks with discrete and distributed delays," IEEE Transactions on Neural Networks, vol. 21, no. 11, pp. 1842-1847, 2010.

[41] J. Qiu, G. Feng, and H. Gao, "Fuzzy-model-based piecewise $H_{\infty}$ static-output-feedback controller design for networked nonlinear systems," IEEE Transactions on Fuzzy Systems, vol. 18, no. 5, pp. 919-934, 2010. 


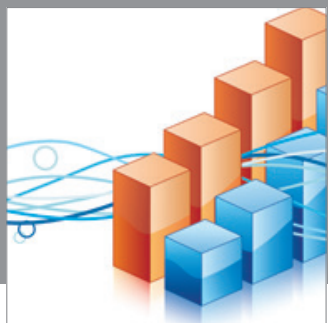

Advances in

Operations Research

mansans

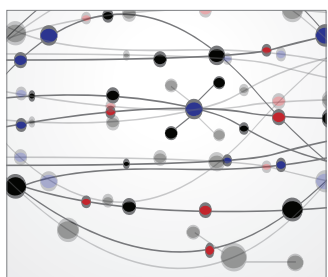

The Scientific World Journal
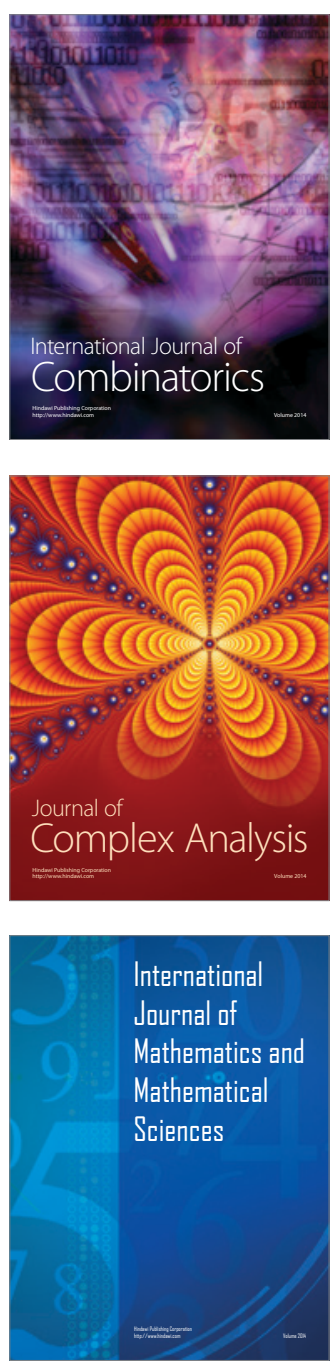
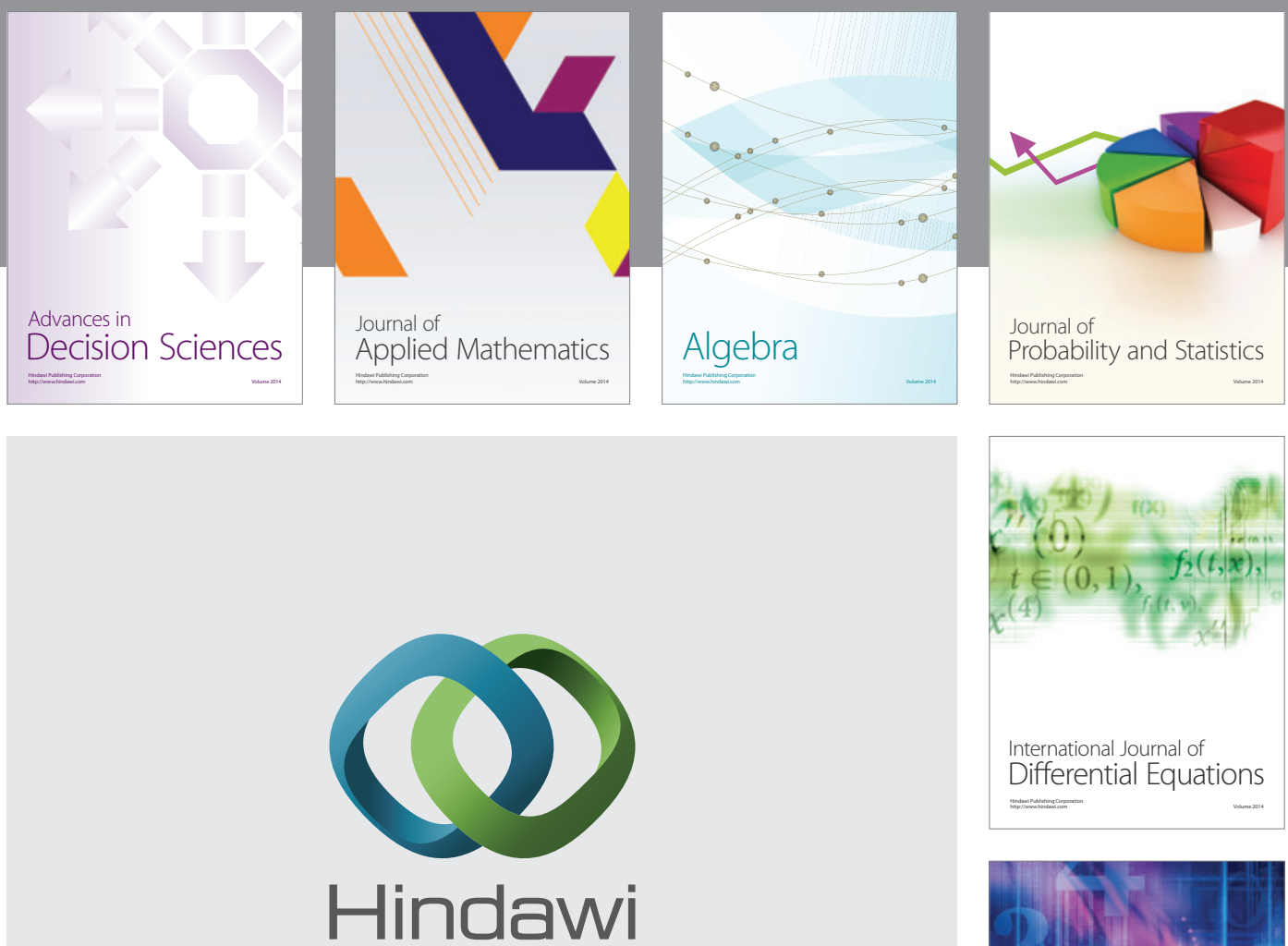

Submit your manuscripts at http://www.hindawi.com
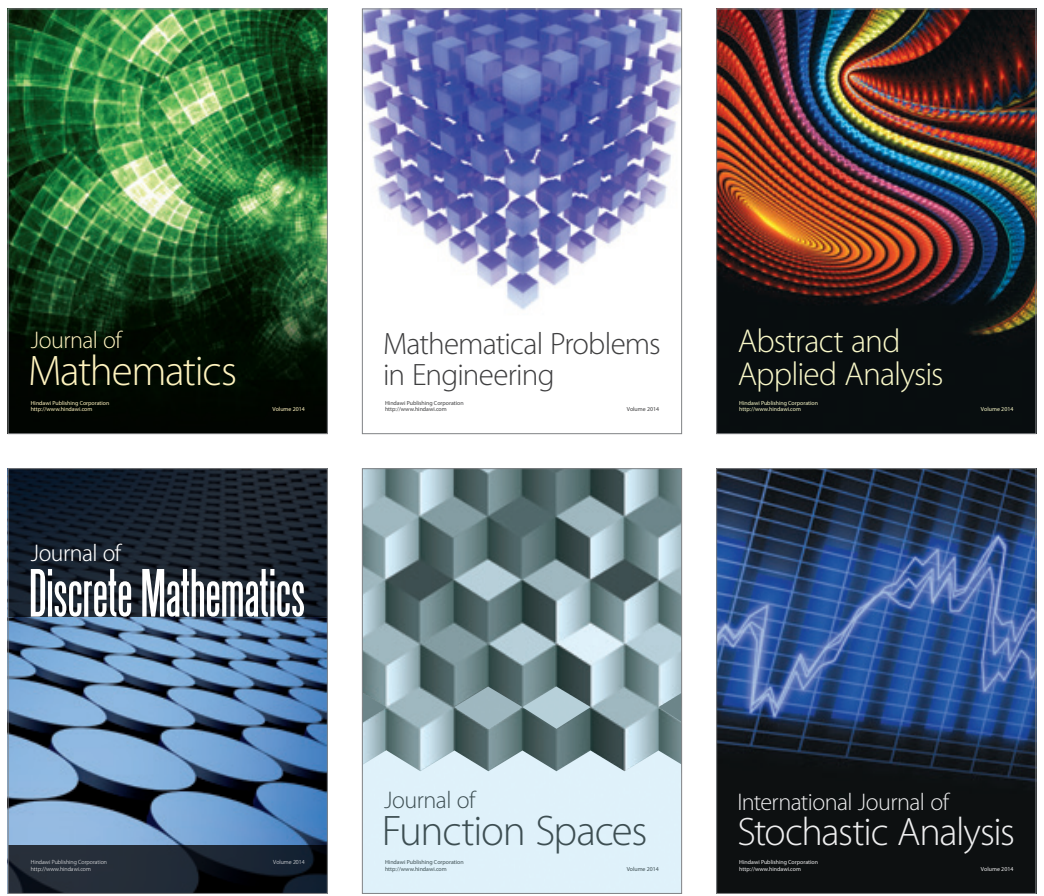

Journal of

Function Spaces

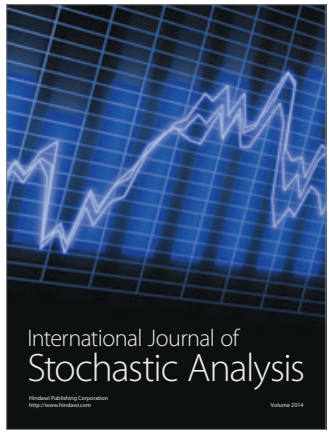

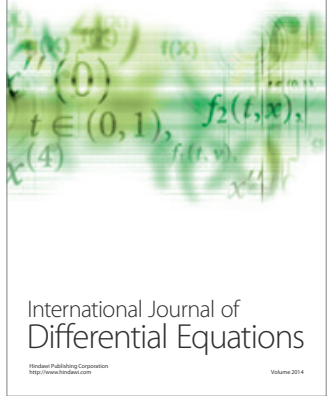
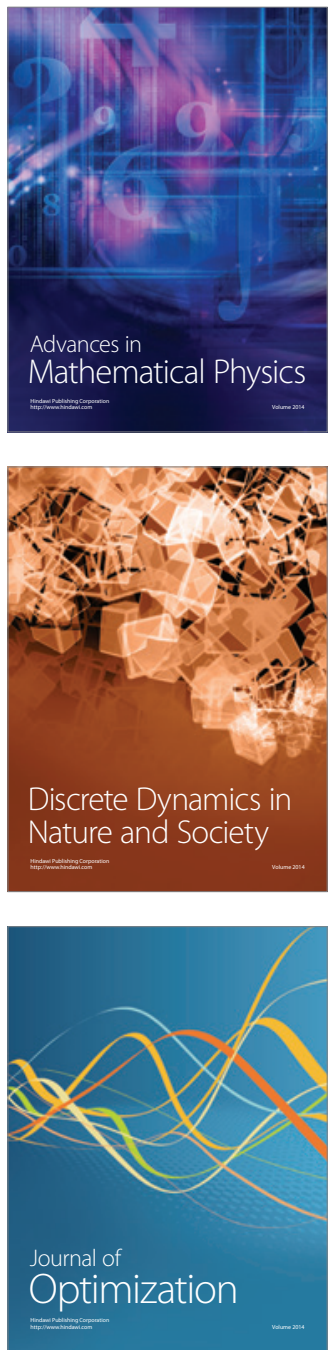